\title{
THE ANALYTICAL SOLUTION OF TELEGRAPH EQUATION OF SPACE-FRACTIONAL ORDER DERIVATIVE BY THE ABOODH TRANSFORM METHOD
}

\author{
MOHAMED ELARBI BENATTIA ${ }^{1, *}$, KACEM BELGHABA ${ }^{2}$ \\ ${ }^{1}$ High School of Economic, Laboratory of Mathematics and Its Applications (LAMAP), Oran, Algeria \\ ${ }^{2}$ University of Oran 1, Laboratory of Mathematics and Its Applications (LAMAP), Oran, Algeria \\ *Corresponding author: mohamed.benattia74@yahoo.com
}

\begin{abstract}
In this article, an analytical solution based on the series expansion method is proposed to solve the telegraph equation of space - fractional order (TESFO), namely the Aboodh transformation method (ATM) subjected to the appropriate initial condition. Using ATM, it is possible to find exact solution or a closed approximate solution of a differential equation. Finally, several numerical examples are given to illustrate the accuracy and stability of this method.
\end{abstract}

\section{INTRODUCTION}

In the last few decades, fractional calculus found many applications in various fields of physical sciences such as viscoelasticity, diffusion, control, relaxation processes and so on [1]. Suspension flows are traditionally modeled by parabolic partial differential equations. Sometimes they can be better modeled by hyperbolic equations such as the telegraph equation, which have parabolic asymptotic. In particular the experimental data described in [1] seem to be better modeled by the telegraph equation than by the heat equation. The telegraph equation is used in signal analysis for transmission and propagation of electrical signals and also used modeling reaction diffusion. The different type solutions of the fractional telegraph equations have been

Received 2019-12-22; accepted 2020-01-20; published 2020-03-02.

2010 Mathematics Subject Classification. 65R10, 26A33.

Key words and phrases. Aboodh Transform; fractional differential equation; Caputo fractional derivative; telegraph equation. (C)2020 Authors retain the copyrights of their papers, and all open access articles are distributed under the terms of the Creative Commons Attribution License. 
discussed by Momani [2] by using decomposition method, Yildirim [3] by homotopy perturbation method. Our concern in this work is to consider the space-fractional telegraph equations as

$$
D_{x}^{\alpha} u(x, t)=a u_{t}+u_{t t}+b u(x, t)+g(x, t), 0<x<1
$$

where $t \geq 0,0<\alpha \leq 2, a, b$ are given constants, $g(x, t)$ is given function.

The main objective of this paper is to introduce a new analytical and approximate solution of spatial fractional telegraphic equations using the Aboodh transformation method(ATM), where in [5] authors proposed a Sumudu transformation method (STM) which is used to solve this equation.

\section{Preliminary}

2.1. Fundamental Properties of Fractional Calculus. In this section we give definitions and some basic results.

Definition 2.1. An Aboodh transform is defined for functions of exponential order. We consider functions in the set $F$ defined by;

$$
F=\left\{f ( t ) : | f ( t ) | < M e ^ { - v t } , \text { if } t \in \left[0 ; \infty\left[, M, k_{1}, k_{2}>0 ; k_{1} \leq v \leq k_{2}\right\}\right.\right.
$$

For a given function in the set $F, M$ must be finite number and $k_{1}, k_{2}$ may be finite or infinite with variable $v$ define as $k_{1} \leq v \leq k_{2}$. Then, the Aboodh transform denoted by the operator $A(:)$ is defined by the integral equation:

$$
T(v)=A[f(t)]=\frac{1}{v} \int_{0}^{\infty} f(t) e^{-v t} d t, \quad t \geq 0, k_{1} \leq v \leq k_{2} .
$$

For a given function in the set $F, M$ must be finite number and $k_{1}, k_{2}$ may be finite or infinite with variable $v$ define as $k_{1} \leq v \leq k_{2}$. Then, the Aboodh transform denoted by the operator $A(:)$ is defined by the integral equation:

$$
T(v)=A[f(t)]=\frac{1}{v} \int_{0}^{\infty} f(t) e^{-v t} d t, \quad t \geq 0, k_{1} \leq v \leq k_{2} .
$$

Standard Aboodh transform for some special functions found are given below in Table (2.1). 


\begin{tabular}{|c|c|}
\hline$f(t)$ & $T(v)=A[f(t)]$ \\
\hline 1 & $\frac{1}{v^{2}}$ \\
\hline$t$ & $\frac{1}{v^{3}}$ \\
\hline$t^{n}, n \geq 1$ & $\frac{n !}{v^{n+2}}$ \\
\hline$e^{a t}$ & $\frac{1}{v^{2}-a v}$ \\
\hline $\sin (a t)$ & $\frac{1}{v\left(v^{2}+a^{2}\right)}$ \\
\hline $\cos (a t)$ & $\frac{1}{v^{2}+a^{2}}$ \\
\hline $\sinh (a t)$ & $\frac{1}{v\left(v^{2}-a^{2}\right)}$ \\
\hline$t \cosh (a t)$ & $\frac{1}{v^{2}-a^{2}}$ \\
\hline
\end{tabular}

TABLE(2.1): Aboodh transform of some functions.

Definition 2.2. The Riemann-Liouville fractional integral of order $\alpha \in R^{+}$is defined as

$$
\begin{gathered}
D^{-\alpha} f(t)=I^{\alpha} f(t)=\frac{1}{\Gamma(\alpha)} \int_{0}^{t} \frac{f(x)}{(t-x)^{1-\alpha}} d x, 0<\alpha \leq 1 \\
I^{0} f(t)=f(t)
\end{gathered}
$$

Properties of the operator $I^{\alpha}$ can be found in for $\alpha, \beta \geqslant 0$, and $\gamma \geqslant-1$, we have:

$$
\begin{aligned}
I^{\alpha} I^{\beta} f(t) & =I^{\beta} I^{\alpha} f(t)=I^{\alpha+\beta} f(t) \\
I^{\alpha} t^{\gamma} & =\frac{\Gamma(\gamma+1)}{\Gamma(\alpha+\gamma+1)} t^{\alpha+\gamma}
\end{aligned}
$$

Definition 2.3. The caputo fractional derivative (CFD) operator $D_{t}^{\alpha}$ of order $\alpha$ is

$$
D_{t}^{\alpha} f(t)=I^{n-\alpha} D^{n} f(t)=\frac{1}{\Gamma(n-\alpha)} \int_{0}^{t} \frac{f^{(n)}(x)}{(t-x)^{1+\alpha-n}} d x, x>0
$$

for $n-1<\alpha \leq n, n \in \mathbb{N}, t>0$.

Definition 2.4. The Mittage Leffler function $E_{\alpha}(z)$ with $\alpha>0$, is definite by the following series:

$$
E_{\alpha}(z)=\frac{z^{\alpha}}{\Gamma(n \alpha+1)}, z \in \mathbb{C}
$$

where $n \in Z^{+}, \alpha \in R^{+}$.

Definition 2.5. The Aboodh transform $A\left[D_{x}^{\alpha} f(x)\right]$ of the fractional derivative using the Caputo idea of the function is given by:

$$
\left.A\left[D_{x}^{\alpha} f(x)\right]\right]=v^{\alpha} T(v)-\sum_{k=0}^{n-1} \frac{f^{(k)}(0)}{v^{2-\alpha+k}}
$$


It is easy to understand that:

$$
\left.\left.A\left[D_{t}^{\alpha} f(x ; t)\right]\right]=v^{\alpha} A[f(x ; t)]\right]-\sum_{k=0}^{n-1} \frac{f^{(k)}(x ; 0)}{v^{2-\alpha+k}}, n-1<\alpha \leq n,
$$

Remark 2.1. The Aboodh transform is linear, i.e., if $\alpha$ and $\beta$ are any constants and $f(t)$ and $g(t)$ are functions defined over the set $F$ above, then

$$
A[\alpha f(t) \pm \beta g(t)]=\alpha A[f(t)] \pm \beta A[g(t)]
$$

\section{Procedure Solution Using ATM for Solving Linear TeSfo}

We consider the following linear TESFO of the form:

$$
\begin{gathered}
D_{x}^{\alpha} u(x, t)=a u_{t}+u_{t t}+b u(x, t)+g(x, t), 0<x<1, \\
t \geq 0,0<\alpha \leq 2
\end{gathered}
$$

where $g(x, t)$ is the source term and $a, b$ are constants.

With Initial Condition

$$
\frac{\partial^{(r)} u(0, t)}{\partial x^{r}}=\left.u^{(r)}(0, t)\right|_{t=0}=f_{r}(t), r=0,1,2, \ldots \ldots, n-1 .
$$

Now applying the AT into $\mathrm{Eq}(3.1)$ we have:

$$
A\left[D_{x}^{\alpha} u(x, t)\right]=A\left[a u_{t}+u_{t t}+b u(x, t)\right]+A[g(x, t)]
$$

Substituting $\operatorname{Eq}(2.8)$ into $\operatorname{Eq}(3.3)$ we get:

$$
\begin{gathered}
\left.v^{\alpha} A[u(x ; t)]\right]-\sum_{k=0}^{m-1} \frac{u^{(k)}(0 ; t)}{v^{2-\alpha+k}}=A\left[a u_{t}+u_{t t}+b u(x, t)\right]+A[g(x, t)] \\
A[u(x ; t)]=\sum_{k=0}^{m-1} \frac{f_{k}(t)}{v^{2+k}}+v^{-\alpha} A\left[a u_{t}+u_{t t}+b u(x, t)\right]+v^{-\alpha} A[g(x, t)]
\end{gathered}
$$

So, according to Aboodh decomposition method (ADM) we can obtain the solution result $u(x, t)$ as

$$
u(x, t)=\sum_{n=0}^{\infty} u_{n}(x, t)
$$

Now, substituting $\operatorname{Eq}(3.6)$ into $\operatorname{Eq}(3.5)$ gives

$$
A\left[\sum_{n=0}^{\infty} u_{n}(x, t)\right]=\sum_{k=0}^{m-1} \frac{f_{k}(t)}{v^{2+k}}+v^{-\alpha} A\left[a\left(\sum_{n=0}^{\infty} u_{n}(x, t)\right)_{t}+\left(\sum_{n=0}^{\infty} u_{n}(x, t)\right)_{t t}+b \sum_{n=0}^{\infty} u_{n}(x, t)\right]+v^{-\alpha} A[g(x, t)]
$$

From $\mathrm{Eq}(3.7)$ we can define all the coefficients of $u_{n+1}(x, t)$

So we get the zero coefficients $u_{0}(x, t)$ as:

$$
A\left[u_{0}(x, t)\right]=\sum_{k=0}^{m-1} \frac{f_{k}(t)}{v^{2+k}}
$$


The first component $u_{1}(x, t)$ as:

$$
A\left[u_{1}(x, t)\right]=v^{-\alpha} A\left[a\left(u_{0}(x, t)\right)_{t}+\left(u_{0}(x, t)\right)_{t t}+b u_{0}(x, t)+g(x, t)\right]
$$

Finally the remaining coefficients of $u_{n+1}(x, t)$ can be find in a way like each coefficients is found by using the coming before components.

$$
A\left[u_{n+1}(x, t)\right]=v^{-\alpha} A\left[a\left(u_{n}(x, t)\right)_{t}+\left(u_{n}(x, t)\right)_{t t}+b u_{n}(x, t)+g(x, t)\right], n \geq 0 .
$$

Applying the Aboodh inverse to the above equations yields the following:

$$
\begin{gathered}
u_{0}(x, t)=A^{-1}\left[\sum_{k=0}^{m-1} \frac{f_{k}(t)}{v^{2+k}}\right] \\
u_{1}(x, t)=A^{-1}\left[v^{-\alpha} A\left[a\left(u_{0}(x, t)\right)_{t}+\left(u_{0}(x, t)\right)_{t t}+b u_{0}(x, t)+g(x, t)\right]\right] \\
\vdots \\
u_{n+1}(x, t)=A^{-1}\left[v^{-\alpha} A\left[a\left(u_{n}(x, t)\right)_{t}+\left(u_{n}(x, t)\right)_{t t}+b u_{n}(x, t)+g(x, t)\right]\right]
\end{gathered}
$$

So that, the $\operatorname{AS} u_{n}(x, t)$ is given as:

$$
u_{n}(x, t)=\sum_{j=0}^{n-1} u_{j}(x, t)
$$

Such that

$$
\lim _{n \rightarrow \infty} u_{n}(x, t)=u(x, t)
$$

\section{Illustrative Examples}

In this section we shall test two examples using the ATM to solve the TESFO and the solutions we got it by using the present procedure will be comparing with original ES.

Example 4.1. consider the following homogeneous TESFO

$$
D_{x}^{\alpha} u(x, t)=u_{t t}+u_{t}+u, \quad x, t \geq 0,0<\alpha \leq 2,
$$

with initial conditions

$$
\left\{\begin{array}{l}
u(0, t)=e^{-t}, t \geq 0 \\
u_{x}(0, t)=e^{-t}, t \geq 0
\end{array}\right.
$$

we appling the AT with (2.8) into (4.1) and (4.2) we get:

$$
v^{\alpha} A[u(x ; t)]-\sum_{k=0}^{1} \frac{u^{(k)}(0 ; t)}{v^{2-\alpha+k}}=A\left[u(x, t)_{t t}+u(x, t)_{t}+u(x, t)\right]
$$

So, we have

$$
A[u(x ; t)]=\frac{e^{-t}}{v^{2}}+\frac{e^{-t}}{v^{3}}+v^{-\alpha} A\left[u(x, t)_{t t}+u(x, t)_{t}+u(x, t)\right]
$$


So, according to $\mathrm{ADM}$ we can obtain the solution result $u(x, t)$ as

$$
u(x, t)=\sum_{n=0}^{\infty} u_{n}(x, t)
$$

substituting (3.6) into (4.4) gives

$$
\left.\left.A\left[\sum_{n=0}^{\infty} u_{n}(x, t)\right]=\frac{e^{-t}}{v^{2}}+\frac{e^{-t}}{v^{3}}+v^{-\alpha} A\left[\left(\sum_{n=0}^{\infty} u_{n}(x, t)\right)\right)_{t t}+\left(\sum_{n=0}^{\infty} u_{n}(x, t)\right)\right)_{t}+\left(\sum_{n=0}^{\infty} u_{n}(x, t)\right)\right]
$$

according to equation (4.5), we can calculate the terms $u_{n+1}(x, t)$

So, we get the coefficients of $u_{0}(x, t)$ as

$$
A\left[u_{0}(x ; t)\right]=\frac{e^{-t}}{v^{2}}+\frac{e^{-t}}{v^{3}}
$$

So, we can use the Aboodh inverse in (4.6), we get

$$
u_{0}(x ; t)=A^{-1}\left[\frac{e^{-t}}{v^{2}}+\frac{e^{-t}}{v^{3}}\right]=e^{-t}+x e^{-t}
$$

and in the same way we calculate the coefficients of $u_{1}(x, t)$

$$
A\left[u_{1}(x, t)\right]=v^{-\alpha} A\left[\left(u_{0}(x, t)\right)_{t t}+\left(u_{0}(x, t)\right)_{t}+\left(u_{0}(x, t)\right)\right]
$$

Also, we have

$$
\begin{aligned}
& u_{1}(x, t)=A^{-1}\left[v^{-\alpha} A\left[\left(u_{0}(x, t)\right)_{t t}+\left(u_{0}(x, t)\right)_{t}+\left(u_{0}(x, t)\right)\right]\right] \\
& \left\{\begin{array}{l}
u_{1}(x, t)=A^{-1}\left[v^{-\alpha} A\left[e^{-t}+x e^{-t}\right]\right] \\
=A^{-1}\left[\frac{e^{-t}}{v^{\alpha}}+\frac{e^{-t}}{v^{\alpha+3}}\right]=e^{-t} A^{-1}\left[\frac{1}{v^{\alpha+2}}+\frac{1}{v^{\alpha+3}}\right] \\
=e^{-t}\left(\frac{x^{\alpha}}{\Gamma(\alpha+1)}+\frac{x^{\alpha+1}}{\Gamma(\alpha+2)}\right)
\end{array}\right.
\end{aligned}
$$

We can find the coefficients of $u_{n}(x, t)$ with the recurente relation as follows

$$
u_{n+1}(x, t)=A^{-1}\left[v^{-\alpha} A\left[\left(u_{n}(x, t)\right)_{t t}+\left(u_{n}(x, t)\right)_{t}+\left(u_{n}(x, t)\right)\right]\right], \forall n \geq 0
$$

Also, we have

$$
\begin{gathered}
u_{2}(x, t)=e^{-t}\left(\frac{x^{2 \alpha}}{\Gamma(2 \alpha+1)}+\frac{x^{2 \alpha+1}}{\Gamma(2 \alpha+2)}\right) \\
\vdots \\
\vdots \\
u_{n}(x, t)=e^{-t}\left(\frac{x^{n \alpha}}{\Gamma(n \alpha+1)}+\frac{x^{n \alpha+1}}{\Gamma(n \alpha+2)}\right)
\end{gathered}
$$


Finally, we obtain the approximate solution

$$
u_{n}(x, t)=e^{-t}\left(1+x+\frac{x^{\alpha}}{\Gamma(\alpha+1)}+\frac{x^{\alpha+1}}{\Gamma(\alpha+2)}+\frac{x^{2 \alpha}}{\Gamma(2 \alpha+1)}+\frac{x^{2 \alpha+1}}{\Gamma(2 \alpha+2)}+\ldots \ldots \ldots \ldots \ldots \ldots . . .\right)
$$

If we put $\alpha=1 \mathrm{in}(4.12)$, we can conclude the exact solution

$$
u(x, t)=e^{-t}\left(1+x+x+\frac{x^{2}}{2 !}+\frac{x^{2}}{2 !}+\frac{x^{3}}{3 !}+\frac{x^{3}}{3 !}+\ldots \ldots \ldots \ldots \ldots \ldots\right)=2 e^{-t+x}-e^{-t}
$$

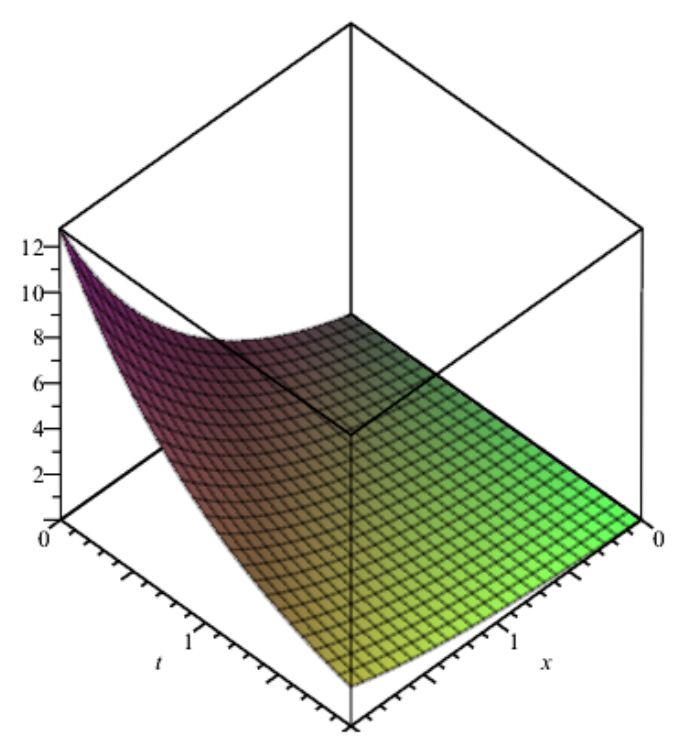

(A) (1.1)

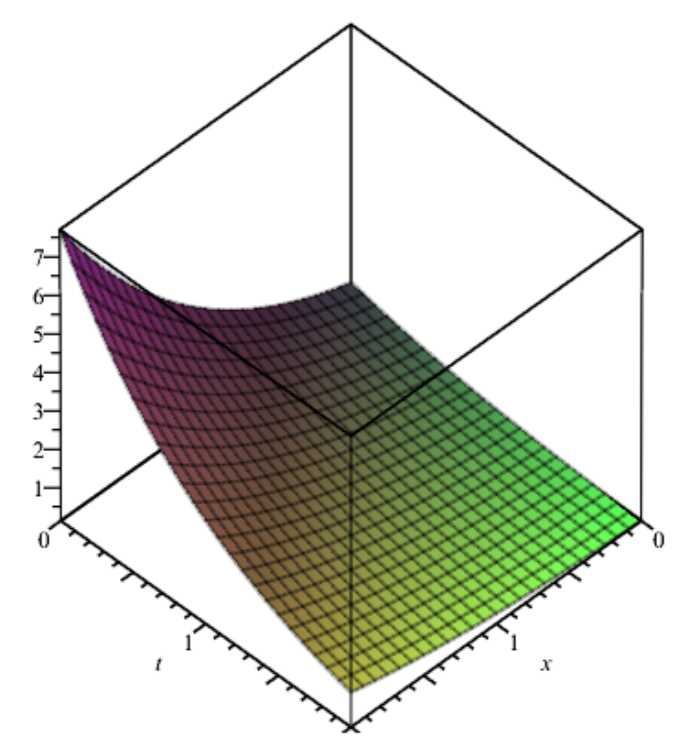

(в) $(1.2)$

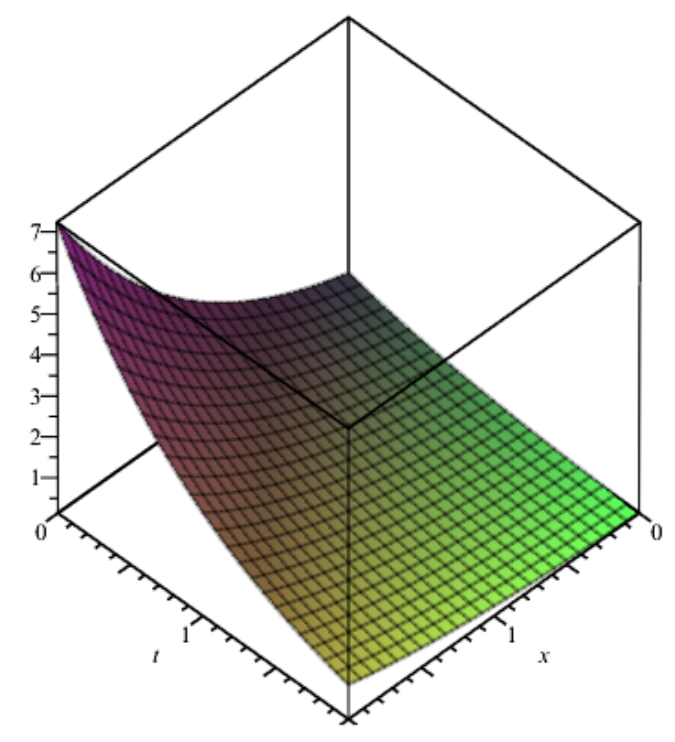

(C) (1.3)

Figure 1. Comparison between (1.1) the exact solution for $\alpha=1$ and (1.2), (1.3) the approximative solutions using 4-term of the ATM for $\alpha=1.7$ and $\alpha=1.9$ respectively. 
Example 4.2. We consider a linear telegraph equation described by

$$
D_{x}^{\alpha} u(x, t)=u_{t t}+2 u_{t}+u \quad x, t \geq 0,0<\alpha \leq 2,
$$

with initial conditions

$$
\left\{\begin{array}{c}
u(0, t)=e^{-3 t}, t \geq 0 \\
u_{x}(0, t)=2 e^{-3 t}, t \geq 0
\end{array}\right.
$$

we appling the AT with (2.8) into (4.13) and (4.14) we get:

$$
v^{\alpha} A[u(x ; t)]-\sum_{k=0}^{1} \frac{u^{(k)}(0 ; t)}{v^{2-\alpha+k}}=A\left[u(x, t)_{t t}+2 u(x, t)_{t}+u(x, t)\right]
$$

So, we have

$$
A[u(x ; t)]=\frac{e^{-3 t}}{v^{2}}+2 \frac{e^{-3 t}}{v^{3}}+v^{-\alpha} A\left[u(x, t)_{t t}+2 u(x, t)_{t}+u(x, t)\right]
$$

So, according to $A D M$ we can obtain the solution result $u(x ; t)$ as

$$
u(x, t)=\sum_{n=0}^{\infty} u_{n}(x, t)
$$

substituting (3.6) into (4.16) gives

$$
\left.\left.A\left[\sum_{n=0}^{\infty} u_{n}(x, t)\right]=\frac{e^{-3 t}}{v^{2}}+2 \frac{e^{-3 t}}{v^{3}}+v^{-\alpha} A\left[\left(\sum_{n=0}^{\infty} u_{n}(x, t)\right)\right)_{t t}+2\left(\sum_{n=0}^{\infty} u_{n}(x, t)\right)\right)_{t}+\left(\sum_{n=0}^{\infty} u_{n}(x, t)\right)\right]
$$

according to equation (4.18), we can calculate the terms $u_{n+1}(x, t)$.

So, we get the coefficients of $u_{0}(x, t)$ as

$$
A\left[u_{0}(x ; t)\right]=\frac{e^{-3 t}}{v^{2}}+2 \frac{e^{-3 t}}{v^{3}}
$$

we use the Aboodh inverse in (4.19), we obtain

$$
u_{0}(x ; t)=e^{-3 t}+2 x e^{-3 t}
$$

and in the same way we calculate the coefficients of $u_{1}(x, t)$

$$
A\left[u_{1}(x, t)\right]=v^{-\alpha} A\left[\left(u_{0}(x, t)\right)_{t t}+2\left(u_{0}(x, t)\right)_{t}+\left(u_{0}(x, t)\right)\right]
$$

Also, we have

$$
u_{1}(x, t)=A^{-1}\left[v^{-\alpha} A\left[\left(u_{0}(x, t)\right)_{t t}+2\left(u_{0}(x, t)\right)_{t}+\left(u_{0}(x, t)\right)\right]\right]
$$




$$
\left\{\begin{array}{c}
u_{1}(x, t)=A^{-1}\left[v^{-\alpha} A\left[4 e^{-3 t}+8 x e^{-3 t}\right]\right] \\
=e^{-3 t} A^{-1}\left[\frac{4}{v^{\alpha+2}}+\frac{8}{v^{\alpha+3}}\right] \\
=4 e^{-3 t}\left(\frac{x^{\alpha}}{\Gamma(\alpha+1)}+\frac{2 x^{\alpha+1}}{\Gamma(\alpha+2)}\right)
\end{array}\right.
$$

We can find the coefficients of $u_{n}(x, t)$ with the recurrence relation as follows

$$
u_{n+1}(x, t)=A^{-1}\left[v^{-\alpha} A\left[\left(u_{n}(x, t)\right)_{t t}+2\left(u_{n}(x, t)\right)_{t}+\left(u_{n}(x, t)\right)\right]\right]
$$

Also, we have

$$
\left\{\begin{array}{c}
u_{2}(x, t)=A^{-1}\left[v^{-\alpha} A\left[\left(u_{1}(x, t)\right)_{t t}+2\left(u_{1}(x, t)\right)_{t}+\left(u_{1}(x, t)\right)\right]\right] \\
=4 A^{-1}\left[v^{-\alpha} A\left[e^{-3 t}\left(\frac{4 x^{\alpha}}{\Gamma(\alpha+1)}+\frac{8 x^{\alpha+1}}{\Gamma(\alpha+2)}\right)\right]\right] \\
=4 e^{-3 t} A^{-1}\left[\frac{4}{v^{2 \alpha+2}}+\frac{8}{v^{2 \alpha+3}}\right] \\
=16 e^{-3 t}\left(\frac{x^{2 \alpha}}{\Gamma(2 \alpha+1)}+\frac{2 x^{2 \alpha+1}}{\Gamma(2 \alpha+2)}\right)
\end{array}\right.
$$

we can give the general solution as follow

$$
u_{n}(x, t)=4^{n} e^{-3 t}\left(\frac{x^{n \alpha}}{\Gamma(n \alpha+1)}+\frac{2 x^{n \alpha+1}}{\Gamma(n \alpha+2)}\right)
$$

Finally, we obtain the approximate solution

$$
u_{n}(x, t)=e^{-3 t}\left(1+2 x+\frac{4 x^{\alpha}}{\Gamma(\alpha+1)}+\frac{8 x^{\alpha+1}}{\Gamma(\alpha+2)}+\frac{16 x^{2 \alpha}}{\Gamma(2 \alpha+1)}+\frac{32 x^{2 \alpha+1}}{\Gamma(2 \alpha+2)}+\ldots \ldots \frac{4^{n} x^{n \alpha}}{\Gamma(n \alpha+1)}+2 \frac{4^{n} x^{n \alpha+1}}{\Gamma(n \alpha+2)}+\ldots . .\right)
$$

If we put $\alpha=2$ in (4.26), we can conclude the exact solution

$$
u(x, t)=e^{-3 t}\left(1+2 x+\frac{4 x^{2}}{2 !}+\frac{8 x^{3}}{3 !}+\frac{16 x^{4}}{4 !}+\ldots .\right)=e^{-3 t} e^{2 x}=e^{-3 t+2 x}
$$




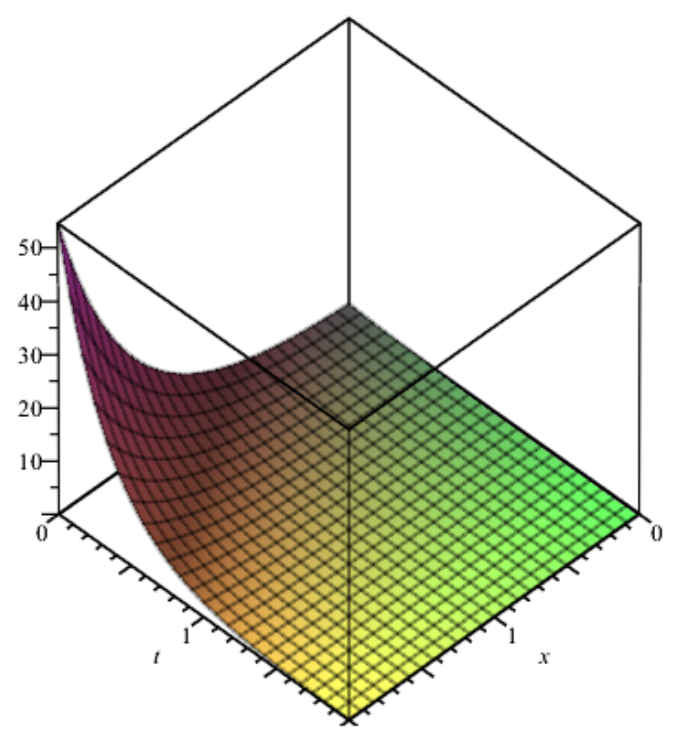

(A) $(2.1)$

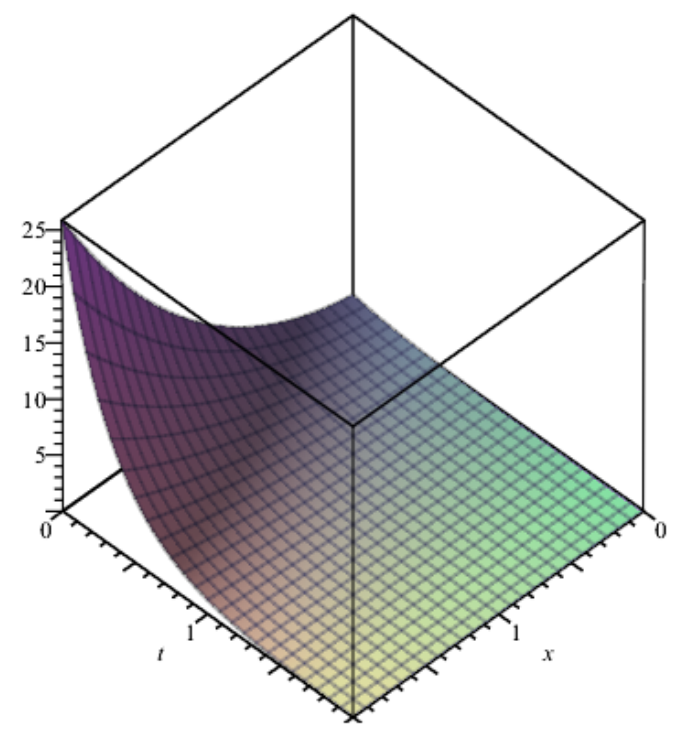

(B) $(2.2)$

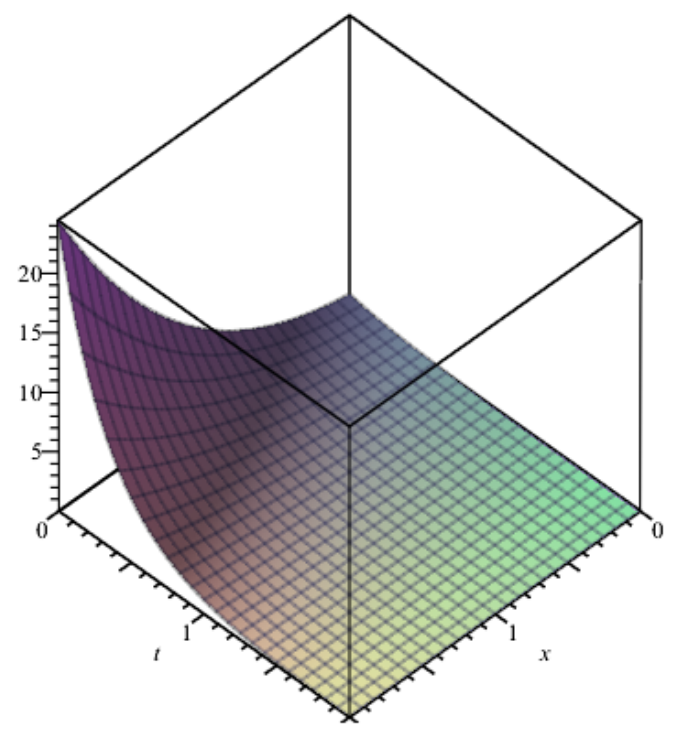

(C) $(2.3)$

Figure 2. Comparison between (2.1) the exact solution for $\alpha=2$ and (2.2), (2.3) the approximative solutions using 4-term of the ATM for $\alpha=1.7$ and $\alpha=1.9$ respectively.

Conclusion. The application of ATM was extended successfully for solving the TESFO. The ATM was clearly very efficient and powerful technique in finding the approximative solution of the proposed equations. In order to check the effectiveness of the introduced procedure, two numerical examples are tested, by comparing the approximative solution with the exact solution.

Conflicts of Interest: The author(s) declare that there are no conflicts of interest regarding the publication of this paper. 


\section{REFERENCES}

[1] S. Kumar. A new analytical modelling for fractional telegraph equation via Laplace transform. Appl. Math. Model. 38 (13) (2014), 3154-3163.

[2] S. Momani. Analytic and approximate solutions of space and time fractional telegraph equations. Appl. Math. Comput., 170 (2005), 1126-1134.

[3] A. Yildirim. He's homotopy perturbation method for solving the space- and time-fractional telegraph equations. Int. J. Comput. Math. 87 (13) (2010), 2998-3006.

[4] M. ELARBI BENATtiA, K. BELGHABA. Numerical Solution for Solving Fractional Differential Equations using Shifted Chebyshev Wavelet. Gen. Lett. Math. 3 (2) (2017), 101-110.

[5] Mohammed G. S. AL-Safi, Wurood R. Abd AL-Hussein, Ayad Ghazi Naser Al-Shammari. A new approximate solution for the Telegraph equation of space-fractional order derivative by using Sumudu method. Iraqi J. Sci. 59 (3A) (2018), 1301-1311.

[6] Mohammed AL-Safi, G.S., Farah,L.J. and Muna, S.A. Numerical Solution for Telegraph Equation of Space Fractional Order using Legendre Wavelets Spectral tau Algorithm, Aust. J. Basic Appl. Sci. 10 (12) (2016), 383-391.

[7] Gupta, V.G., Sharma, B. and Kilicman, A. A Note on Fractional Sumudu Transform. Journal of Applied Mathematics, 2010 (2010), Article ID 154189.

[8] Sunil, K. 2013. A New Analytical Modelling for Fractional Telegraph Equation Via Laplace Transform. Appl. Math. Model. 38 (13) (2014), 3154-3163.

[9] Abdelbagy A. Alshikh, Mohand M. Abdelrahim Mahgob. A Comparative Study Between Laplace Transform and Two New Integrals "ELzaki" Transform and "Aboodh" Transform. Pure Appl. Math. J. 5 (5) (2016), 145-150. 